\title{
DESNUTRICIÓN EN PACIENTES RECIÉN DIAGNOSTICADAS CON CÁNCER GINECOLÓGICO.
}

\author{
UNDERNUTRITION IN NEWLY DIAGNOSED PATIENTS WITH GYNAECOLOGICAL CANCER.
}

\author{
Brito-Alvarez Marisol' ${ }^{1}$, Romero-Lagunes María Luisa², Hernández-Barajas David², Tavitas-Herrera Silvia \\ Elvira², Martínez-González Gustavo Israel ${ }^{1}$.
}

1 Facultad de Salud Pública y Nutrición, UANL. 2 Hospital Universitario Dr. José Eleuterio González, UANL. México.

\section{RESUMEN}

Introducción. La incidencia y prevalencia del cáncer ginecológico en nuestro país continúa creciendo y con ello la desnutrición y sus complicaciones, la valoración global subjetiva generada por el paciente (VGS-GP) es una herramienta que identifica a los pacientes en riesgo de desnutrición/desnutrición moderada y desnutrición severa (Categoría B/C) que requieren intervención nutricional. Objetivo: Determinar la desnutrición que presentan las pacientes recién diagnosticadas con cáncer ginecológico. Material y Método: Estudio transversal, exploratorio, descriptivo, correlacional que incluyó 64 pacientes $>20$ años recién diagnosticadas con cáncer cervicouterino $(\mathrm{CaCu})$, endometrio $(\mathrm{CaE})$, ovario $(\mathrm{CaO})$ y vulvar $(\mathrm{CaV})$, sin tratamiento. Se evaluó mediante la VGS-GP, albúmina, \% pérdida de peso (\%PP). Los datos fueron recopilados en Excel 2011 y analizados en IBM Statistics 23. Resultados: La VGS-GP reportó $25 \%$ de los pacientes en (Categoría B) y $29.7 \%$ (Categoría C). El IMC identificó con desnutrición a 6.3\%. El \%PP en 1 y 6 meses fue mayor en la Categoría B/C (p 0.000), la VGS-GP en categoría B y C presentó mayor correlación con incremento de \%PP, albúmina disminuida y sintomatología en el paciente oncológico. Conclusión: La VGS-GP reportó $29.7 \%$ en desnutrición mientras el IMC el $6.3 \%$, esta herramienta identifica a pacientes con cáncer ginecológico en riesgo de desnutrición/desnutrición moderada y desnutrición severa (54.68\%) que requieren intervención nutricional.

Palabras Clave: Desnutrición, cáncer ginecológico, valoración global subjetiva generada por el paciente.

\section{ABSTRACT}

Introduction: The incidence and prevalence of gynecological cancer in our country continues to grow and with it malnutrition and its complications, the Patient-Generated Subjective Global Assessment (PG-SGA) is a tool that identifies patients at risk of malnutrition/moderate malnutrition and severe malnutrition (Category $\mathrm{B} / \mathrm{C}$ ) that requires nutritional intervention. Objective: To determine the of malnutrition in patients newly diagnosed with gynecological cancer. Material and method: Crosssectional, exploratory, descriptive, correlational study that included 64 patients $>20$ years newly diagnosed with cervical cancer $(\mathrm{CaCu})$, endometrium $(\mathrm{CaE})$, ovary $(\mathrm{CaO})$ and vulvar $(\mathrm{CaV})$, without treatment. We evaluated PG-SGA, albumin, \% weight loss (\%PP). Data collected in Excel 2011 and analyzed in IBM Statistics 23. Results: PG-SGA reported 25\% of patients in (Category B) and 29.7\% (Category C). BMI identified 6.3\% with malnutrition. The percentage PP in 1 and 6 months was higher in Category B/C ( $p 0.000)$, the PG-SGA in category B and C presented greater correlation with increase of \%PP, decreased albumin and symptomatology in the oncological patient. Conclusions: The PG-SGA reported $29.7 \%$ in malnutrition while the $\mathrm{BMI}$ reported $6.3 \%$, this tool identifies patients with gynecological cancer at risk of malnutrition and/or malnutrition $(54.68 \%)$ who require nutritional intervention.

Key words: Undernutrition, gynecological cancer, Patient-Generated Subjective Global Assessment.

Correspondencia: Marisol Brito-Álvarez mari solbrito123@hotmail.com

Recibido: 25 de enero 2021, aceptado: 26 de mayo 2021

(C) Autor2021

(c) (i)

DOI: https://doi.org/10.29105/respyn20.3-4

Citation: Brito-Alvarez M., Romero-Lagunes M.L., Hernández-Barajas D., Tavitas-Herrera S.E., Martínez-

González G.I. (2021) Desnutrición en pacientes recién diagnosticadas con cáncer ginecológico. Revista

Salud Pública y Nutrición, 20 (3), 26-35. 


\section{Introducción}

La Agencia Internacional para la Investigación sobre el Cáncer en su proyecto Globocan 2020, reportó que para el 2020 a nivel internacional en el sexo femenino el número de nuevos casos fue de 9.2 millones, siendo más frecuente el cáncer de mama $24.5 \%$, colo-rectal $9.4 \%$, pulmón $8.4 \%$ y cervicouterino con 6.5\%, (International Agency for Research on Cancer, 2020).

A nivel nacional para el 2020 en el sexo femenino, se reportaron 105,963 casos nuevos, de los cuales un $8.9 \%$ está representado por el cáncer cervicouterino y un $5.2 \%$ por el cáncer de cuerpo de útero; con una incidencia de 12.6 por cada 100, 000 habitantes para cáncer cervicouterino y 7.6 para cáncer de cuerpo de útero, con una mortalidad de 5.7 y 1.6 respectivamente por cada 100,000 habitantes (International Agency for Research on Cancer, 2020).

La desnutrición es una enfermedad a la cual se enfrentan la mayoría de los pacientes que viven con cáncer durante el tratamiento oncológico, puede ser causada; por el propio tratamiento, así como por la patología base, entre las terapias que deterioran el estado nutricional se encuentran la quimioterapia, la radiación, la inmunoterapia, la terapia blanca y el tratamiento quirúrgico (Marian et al, 2010).

La pérdida de tejido muscular en el paciente oncológico es mediada por un incremento del catabolismo, que aumenta el flujo del nitrógeno desde el músculo esquelético al hígado, disminuyendo el suministro plasmático de los principales precursores de la producción de aminoácidos de cadena larga, siendo estos necesarios para estimular la síntesis proteica, el exceso de síntesis de citoquinas pro inflamatorias como IL-1, IL-2, TNF- $\alpha$, que activan el NF-kB que favorece la disminución de síntesis proteica e induce proteólisis (Valenzuela-Landaeta et al, 2012).

La evaluación del estado nutricional de los pacientes oncológicos debe de realizarse desde el inicio y durante el tratamiento; para lograr identificar aquellos pacientes en riesgo y realizar una adecuada intervención nutricional. La valoración global subjetiva generada por el paciente es una técnica que valora el estado nutricional mediante la pérdida de peso, ingesta dietética, síntomas gastrointestinales presentes, capacidad funcional, estrés metabólico, pérdida de masa muscular y grasa subcutánea, es una herramienta rápida y confiable que permite identificar a los pacientes con cáncer que tienen riesgo de desnutrición o desnutrición y que necesitan intervención nutricia (Martínez Roque, 2007) (Detsky et al, 1987) (Ottery, 1994).

La VSG-GP ha demostrado tener una sensibilidad del 96-98\% y una especificidad del $82-83 \%$ en pacientes con cáncer, así como una correlación inversa entre el estado nutricional identificado por la VSG-GP y la calidad de vida de los pacientes (ValenzuelaLandaeta et al, 2012).

La VGS-GP es un instrumento que a cubierto todos los dominios de las definiciones conceptuales de desnutrición, tal como define la Sociedad Europea de Nutrición Clínica y Metabolismo (ESPEN) y la Sociedad Americana de Nutrición Parenteral y Enteral (ASPEN) (Jager-Wittenaar et al, 2017).

De acuerdo con "Oncology Nutrition Dietetic Practice Group" y "the American Dietetic Association", la VGS-GP es un tamizaje que se puede aplicar como parte de la evolución nutricional del adulto con enfermedad oncológica, demostrando la obtención de datos válidos y confiables tanto en cuidados ambulatorios como en la enfermedad aguda (Bauer et al., 2002) (Thompson et al., 2017).

Existen estudios que avalan el uso de la VSG-GP en población oncológica; desde el 2002 Bauer et al., realizó una investigación comparando la Valoración Global Subjetiva (VGS) con la VGS-GP, demostrando que es un tamizaje valido y eficaz con un $98 \%$ de sensibilidad y un $82 \%$ de especificidad para el diagnóstico y riesgo de desnutrición en el paciente oncológico; otra revisión realizada en distintos hospitales de México recomienda el uso de la VGS-GP para evaluar la desnutrición en pacientes oncológicos (Castillo-Martínez et al., 2018). Un estudio realizado también en México obtuvo la validación del tamizaje en población pediátrica para identificar niños con riesgo de desnutrición o desnutrición en pacientes hospitalizados con reciente diagnóstico de cáncer; sin embargo, esta información no se ha implementado en la población adulta con cáncer ginecológico en nuestro país (Vázquez de la Torre et al., 2017). 
Por lo que el objetivo fue determinar el estado nutricional de las pacientes recién diagnosticadas con cáncer ginecológico mediante la valoración global subjetiva generada por el paciente.

\section{Material y Método}

$\underline{\text { Sujetos }}$

Estudio transversal, exploratorio - descriptivo, correlacional, que incluyó 64 pacientes recién diagnosticadas con cáncer ginecológico que acudieron por primera vez a la consulta al departamento de Oncología del "Hospital Universitario Dr. José Eleuterio González", en Nuevo León en el periodo Julio-noviembre 2018.

Los criterios de Inclusión fueron: pacientes ambulatorias > 20 años de edad con diagnóstico histopatológico inicial de cáncer ginecológico: Cáncer de endometrio, cérvico-uterino, ovario, sarcoma uterino, vaginal y vulvar; se excluyeron pacientes hospitalizadas, embarazadas, con tratamiento sistémico oncológico previamente para esta neoplasia, con extremidades amputadas, que no presentaran diagnóstico de cáncer ginecológico, con condiciones cognitivas que impidan la realización de la VGS-GP; los criterios de eliminación fueron: que no aceptara participar en el estudio. El estudio se basó en un nivel de confianza de $95 \%$ aplicando una formula general, considerando el $5 \%$ de error basados en el estudio de Das et al, 2014, obteniendo una $\mathrm{n}$ de 63 pacientes que fueron seleccionados por conveniencia mediante los criterios de inclusión, exclusión y eliminación definidos para el presente estudio. Se informó a los sujetos sobre el objetivo de la investigación y datos a recolectar. Una vez consintiendo su participación de manera verbal de acuerdo con lo estipulado en el reglamento de la ley general de salud en materia de investigación para la salud se llevó a cabo la evaluación nutricional.

\section{Tamizaje Nutricional}

Se aplicó el tamizaje nutricional Valoración Global Subjetiva - Generada por el Paciente por un licenciado en nutrición; este tamizaje incluye valoración de cambios en el peso (pérdida de peso en las últimas dos semanas y durante el último mes), cambios en la ingesta (mayor consumo de alimentos líquidos o sólidos), síntomas que interfieren en la ingesta (náuseas, vómitos, disgeusia, dolor), capacidad funcional (actividades normales, pasa mucho tiempo en cama o silla), la enfermedad y su relación con el incremento de requerimientos nutricionales, estrés metabólico (fiebre, fármacos), evaluación física (pérdida de grasa subcutánea, atrofia muscular, edema) evaluados de forma subjetiva (Jager-Wittenaar et al, 2017).

Fueron clasificados en: Categoría A (estado nutricional adecuado): sin pérdida de peso o sin retención hídrica reciente, sin déficit o mejora en la ingesta, sin síntomas que tengan impacto en la nutrición, en cuanto a funcionalidad sin afección o mejora reciente significativa y en el examen físico sin déficit o deficiencia crónica con reciente mejoría clínica (Jager-Wittenaar et al, 2017).

Categoría B (Riesgo de desnutrición/desnutrición moderada): con $5 \%$ de pérdida de peso en el último mes o $10 \%$ en 6 meses, sin estabilización de peso, disminución significativa de la ingesta, impacto en los síntomas que afectan la ingesta, deterioro de la funcionalidad y pérdida leve a moderada de masa grasa y/o muscular (Jager-Wittenaar et al, 2017).

Categoría C (Desnutrición severa): $>5 \%$ de pérdida de peso en 1 mes o $>10 \%$ de pérdida de peso en 6 meses, déficit severo en la ingesta, afección severa de los síntomas, deterioro severo en la funcionalidad y signos evidentes de desnutrición (pérdida severa de grasa, músculo y posible edema) de acuerdo a los criterios subjetivos que marca el tamizaje (JagerWittenaar et al, 2017).

Es importante considerar que el tamizaje nutricional tiene un apartado para realizar un puntaje, este no interfiere en el diagnóstico, pero si establece la necesidad de intervención nutricional de acuerdo a los resultados: $0-1$ : No requiere intervención nutricional en este momento. Volver a valorar durante el tratamiento. 2 - 3: Paciente y familiares requieren educación nutricional por parte de especialista en nutrición u otro clínico, con intervención farmacológica según los síntomas y la analítica del paciente. 4 - 8: Requiere intervención de un especialista en nutrición junto con su médico/oncólogo para manejo farmacológico de los síntomas. > 9 Indica una necesidad crítica de mejorar el manejo de los síntomas del paciente y/o intervención nutricional/farmacológica (JagerWittenaar et al, 2017). 
Para evaluar el peso se utilizó báscula Omron BF511 (HBF-511T-E/HBF-511B-E), estadímetro hospitalario, la composición corporal se interpretó de acuerdo a los criterios que propone Omron Healthcare que son basados en "Investigaciones de H. D. McCarthy y col., en International Journal of Obesity, Vol. 30, 2006, y de Gallagher y col., American Journal of Clinical Nutrition, Vol. 72, Sept. 2000", niveles séricos de albúmina de acuerdo a criterios de Kłek, 2015, el \% pérdida de peso según el "Consensus statement: Academy of nutrition and dietetics and American society for parenteral and enteral nutrition: Characteristics recommended for the identification and documentation of adult malnutrition (undernutrition)" (White et al, 2012).

Análisis de los datos

Se recolectó la información en una base datos en Excel 2011, se analizaron en el programa IBM statistics 24 donde se realizaron tablas de frecuencia confrontando desnutrición con resto de variables (tipo de cáncer, albúmina, composición corporal), medias, desviación estándar con un intervalo de confianza al 95\% (IC 95\%), para las comparaciones se utilizó la prueba de T - Student, así como pruebas de bondad de ajuste o de Chi cuadrada Pearson.

\section{Resultados}

Se incluyeron 64 pacientes de sexo femenino recién diagnosticadas con cáncer ginecológico sin tratamiento previo, con un rango de edad de 18 a 87 años (media 52 años). El IMC promedio fue de 30.3 $\mathrm{kg} / \mathrm{m} 2$; mientras que las medias de porcentaje de grasa y músculo fueron de $42.8 \%$ y $24.6 \%$ respectivamente. El tipo de cáncer que predominó en la población estudiada fue el cáncer cervicouterino $(\mathrm{CaCu})$ representado por el $67.2 \%$, seguido por el cáncer de endometrio (17.2\%). Las características de la población estudiada se pueden observar en la tabla 1.
Tabla 1. Características de la población

\begin{tabular}{|c|c|c|c|}
\hline & $\begin{array}{c}\text { Media (SD) } \\
n=64\end{array}$ & $n$ & $\%$ \\
\hline Edad (años) & $52.03(13.5)$ & & \\
\hline Peso (kg) & $72.15(17.19)$ & & \\
\hline Talla (m) & $1.54(0.06)$ & & \\
\hline IMC (kg/m2) & $30.3(6.79)$ & & \\
\hline Porcentaje de Grasa $\left(n=59^{*}\right)$ & $42.8(8.06)$ & & \\
\hline Porcentaje de Músculo & $24.6(3.36)$ & & \\
\hline Circunferencia brazo $\left(n=62^{\star}\right)$ & $30.18(4.92)$ & & \\
\hline Albúmina $\mathrm{mg} / \mathrm{dl}\left(\mathrm{n}=45^{\star}\right)$ & $3.89(0.68)$ & & \\
\hline \multicolumn{4}{|l|}{ Tipo de Cáncer (64) } \\
\hline Ca. Cervicouterino & & 43 & 67.2 \\
\hline Ca. Endometrio & & 11 & 17.2 \\
\hline Ca. Ovario & & 9 & 14 \\
\hline Ca. Vulva & & 1 & 1.6 \\
\hline \multicolumn{4}{|l|}{ Estadio Clínico $(n=64)$} \\
\hline Estadio I & & 19 & 29.7 \\
\hline Estadio II & & 17 & 26.6 \\
\hline Estadio III & & 27 & 42.2 \\
\hline Estadio IV & & 1 & 1.6 \\
\hline
\end{tabular}

La evaluación del estado nutricional con base en el IMC reportó al $50 \%$ de la población con obesidad, el $28.1 \%$ con sobrepeso, un $15.6 \%$ con peso normal y un $6.3 \%$ desnutrición.

En cuanto a la evaluación del estado nutricional con base en la VGS-GP el $45.3 \%$ de las pacientes estaban en "Categoría A - Bien nutrido", el $25 \%$ se encontraba en "Categoría B - Riesgo de desnutrición/desnutrición moderada y un $29.68 \%$ en "Categoría C - Desnutrición severa; en conjunto “Categoría B y C - Riesgo de desnutrición/desnutrición moderada y desnutrición severa representando el $54.68 \%$ de la población.

De acuerdo con el IMC solo el $6.3 \%$ del total de la población estudiada tenía desnutrición comparado con un $29.7 \%$ reportado por la VGS-GP un $29.7 \%$, se puede observar como el IMC subestima el porcentaje de personas en esta situación (Tabla 2). 
Tabla 2. Estado Nutricional de la población evaluada de acuerdo a IMC y VGS-GP

\begin{tabular}{|c|c|c|c|c|c|c|c|c|}
\hline \multirow{4}{*}{ IMC } & \multicolumn{6}{|c|}{ VGS-GP: Categoría } & & \\
\hline & \multirow{2}{*}{\multicolumn{2}{|c|}{$\begin{array}{c}A \\
(n=29,45.3 \%)\end{array}$}} & \multirow{2}{*}{\multicolumn{2}{|c|}{$\begin{array}{c}\text { B } \\
(n=16,25 \%)\end{array}$}} & \multirow{2}{*}{\multicolumn{2}{|c|}{$\begin{array}{c}C \\
(n=19,29.68 \%)\end{array}$}} & \multirow{2}{*}{\multicolumn{2}{|c|}{ Total }} \\
\hline & & & & & & & & \\
\hline & $\mathrm{n}$ & $\%$ & $\mathrm{n}$ & $\%$ & $\mathrm{n}$ & $\%$ & $\mathrm{n}$ & $\%$ \\
\hline Desnutrición & 1 & 3.4 & 0 & 0.0 & 3 & 15.8 & 4 & 6.3 \\
\hline Normopeso & 3 & 10.3 & 3 & 18.8 & 4 & 21.1 & 10 & 15.6 \\
\hline Sobrepeso & 7 & 24.1 & 4 & 25.0 & 7 & 36.8 & 18 & 28.1 \\
\hline Obesidad & 18 & 62.1 & 9 & 56.2 & 5 & 26.3 & 32 & 50.0 \\
\hline Total & 29 & 100.0 & 16 & 100.0 & 19 & 100.0 & 64 & 100.0 \\
\hline
\end{tabular}

La VGS-GP reportó que la prevalencia de pacientes en la categoría bien nutrido era del $45.3 \%$, de las cuales el $55.2 \%$ tenían diagnóstico de $\mathrm{CaCu}$, el 27.6 \% Ca de Endometrio y el $17.2 \%$ Ca de Ovario. Así mismo el $54.7 \%$ de las pacientes presentaba riesgo de desnutrición/desnutrición moderada y desnutrición severa, siendo que el $77.1 \%$ tenían diagnóstico de $\mathrm{CaCu}, 11.4 \%$ Ca de Ovario, $8.6 \% \mathrm{Ca}$ de Endometrio y $2.9 \%$ Ca de Vulva. Al comparar ambos grupos de pacientes se obtuvo que el cáncer cervicouterino presenta mayor incidencia de riesgo de desnutrición/desnutrición moderada y desnutrición severa (p 0.04) (Categoría B y C) (Tabla $3)$.

Tabla 3. Estado nutricional por tipo de cáncer de acuerdo a VGS-GP

\begin{tabular}{lrrrrrr}
\hline \multicolumn{1}{c}{ VGS-GP: Categoría } \\
Tipo de cáncer & \multicolumn{1}{c}{ A } & \multicolumn{3}{c}{ B y C } \\
& $(\mathrm{n}=29,45.3 \%)$ & $(\mathrm{n}=35,54.7 \%)$ & \multicolumn{2}{c}{ Total } \\
& $\mathrm{n}$ & $\%$ & $\mathrm{n}$ & $\%$ & $\mathrm{n}$ & $\%$ \\
\hline CaCU & 16 & 55.2 & 27 & 77.1 & 43 & 67.2 \\
Ca. Endometrio* & 8 & 27.6 & 3 & 8.6 & 11 & 17.2 \\
Ca. Ovario & 5 & 17.2 & 4 & 11.4 & 9 & 14.1 \\
Ca. Vulva* & 0 & 0.0 & 1 & 2.9 & 1 & 1.6 \\
Total & 29 & 100.0 & 35 & 100.0 & 64 & 100.0 \\
\hline Fuente: directa, $\mathrm{n}=64$ & & & & & & \\
*P<.05 & & & & & &
\end{tabular}

$\mathrm{Al}$ analizar los resultados cuantitativos de tamizaje se obtuvo que el $4.7 \%$ obtuvo una puntación de $(0-1)$ por lo que no requirieron intervención nutricional inmediata; el $21.9 \%$ se encontraba en un rango de (2 - 3 puntos), donde tanto paciente como familiar requirieron educación nutricional por especialista en nutrición y abordaje terapéutico de los síntomas; el $32.8 \%$ se situó en un puntaje de $(4-8)$ y requirió intervención nutricional por especialista en nutrición y trabajo de manera coordinada con oncólogo en manejo farmacológico de los síntomas, mientras que el $40.6 \%$ obtuvo puntaje $>9$ y presentó necesidad crítica de mejorar el manejo de los síntomas del paciente y/o intervención nutricional/farmacológica. Respecto a la evaluación de la masa muscular el 51.7 $\%$ de la población que se encontraba bien nutrida presentaba porcentaje de músculo normal y el $44.8 \%$ porcentaje bajo. En la categoría B y C el $34.3 \%$ presentó musculatura normal y un $42.9 \%$ presentó baja musculatura; en cuanto al porcentaje de grasa el $75.9 \%$ de las pacientes con (Categoría A) bien nutrido presentó un porcentaje de grasa muy alto y $48.6 \%$ que presento la misma característica se encontraban en riesgo de desnutrición o desnutrición (Categoría B y $\mathrm{C}$ ) siendo estadísticamente significativo (p 0.0259). Al evaluar al total de la población se obtuvo que el $60.9 \%$ presentó un muy alto porcentaje de grasa, $18.8 \%$ tenía un alto porcentaje, el $10.9 \%$ se encontraba en rangos de normalidad y solamente $1.6 \%$ presentó porcentaje bajo.

El estadio clínico de la enfermedad y su relación con el estado nutricional reportó como resultado que conforme va aumentando el estadio clínico disminuye el porcentaje de pacientes bien nutridas y aumenta el porcentaje riesgo de desnutrición/desnutrición severa, el $44.8 \%$ de las pacientes en etapa I se encontraban en "Categoría A - Bien nutrido" mientras un $17.1 \%$ se encuentro en categoría B y C siendo estadísticamente significativo (p 0.0158), de las pacientes en etapa III el $54.3 \%$ presentó riesgo de desnutrición/desnutrición severa y un $27.6 \%$ se encontraba bien nutrida siendo estadísticamente significativo (p 0.0313). De las pacientes bien nutridas el $44.8 \%$ se encontraba en la etapa I de la enfermedad, $27.6 \%$ en etapa II, $27.6 \%$ en etapa III y no se identificó ninguna en etapa IV. De las pacientes de las categorías B y C el $29.7 \%$ estaba en etapa I, $25.7 \%$ en etapa II, $54.3 \%$ en etapa III y $2.9 \%$ en etapa IV.

Al estudiar la presencia de signos y síntomas que impiden consumo de alimentos de acuerdo a la VGSGP el $20.7 \%$ de las pacientes en categoría A tenían alguna sintomatología contra el $74.3 \%$ de las pacientes con riesgo de desnutrición y desnutrición severa ( $p$ 0.000); en las pacientes con riesgo de desnutrición y desnutrición severa los signos clínicos 
más prevalentes con significancia estadística fueron dolor, la falta de apetito, estreñimiento, náuseas, Alimentos con sabor raro, desagrado de los olores, plenitud y vomito (Tabla 4).

\begin{tabular}{|c|c|c|c|c|c|c|}
\hline \multirow{3}{*}{ Característica Clínica } & \multicolumn{4}{|c|}{ VGS-GP: Categoría } & & \\
\hline & \multicolumn{2}{|c|}{$\begin{array}{c}A \\
(n=29,45.3 \%)\end{array}$} & \multicolumn{2}{|c|}{$\begin{array}{c}\text { B y C } \\
(n=35,54.7 \%)\end{array}$} & \multicolumn{2}{|c|}{ Total } \\
\hline & $\mathrm{n}$ & $\%$ & $n$ & $\%$ & $\mathrm{n}$ & $\%$ \\
\hline Con síntomas & 6 & 20.7 & 26 & 74.3 & 32 & 50.0 \\
\hline Sin problemas & 23 & 79.3 & 9 & 25.7 & 32 & 50.0 \\
\hline Náuseas & 0 & 0.0 & 10 & 28.6 & 10 & 15.6 \\
\hline Vómito & 0 & 0.0 & 5 & 14.3 & 5 & 7.8 \\
\hline Estreñimiento & 3 & 10.3 & 17 & 48.6 & 20 & 31.3 \\
\hline Diarrea & 0 & 0.0 & 4 & 11.4 & 4 & 6.3 \\
\hline Llagas en la boca & 0 & 0.0 & 3 & 8.6 & 3 & 4.7 \\
\hline Sequedad en boca & 2 & 6.9 & 6 & 17.1 & 8 & 12.5 \\
\hline Alimentos con sabor raro & 1 & 3.4 & 9 & 25.7 & 10 & 15.6 \\
\hline Sequedad en boca & 0 & 0.0 & 2 & 5.7 & 2 & 3.1 \\
\hline Alimentos con sabor raro & 1 & 3.4 & 9 & 25.7 & 10 & 15.6 \\
\hline Problemas al tragar & 0 & 0.0 & 2 & 5.7 & 2 & 3.1 \\
\hline Desagrado de olores & 0 & 0.0 & 7 & 20.0 & 7 & 10.9 \\
\hline Plenitud & 1 & 3.4 & 7 & 20.0 & 8 & 12.5 \\
\hline Dolor & 3 & 10.3 & 18 & 51.4 & 21 & 32.8 \\
\hline Otros & 2 & 6.9 & 4 & 11.4 & 6 & 9.4 \\
\hline
\end{tabular}

Cuando se evaluó la sintomatología por tipo de cáncer se observó que las pacientes más sintomáticas eran las diagnosticadas con $\mathrm{CaCU}(55.8 \%)$ y $\mathrm{Ca}$ de Ovario (55.5\%), seguidas de las pacientes con Ca de endometrio (27.3\%). Los signos más frecuentemente reportados por las pacientes con $\mathrm{CaCU}$ fueron el dolor y el estreñimiento (41.8\%), seguidos por la falta de apetito $(32.5 \%)$. En las pacientes con cáncer de endometrio los signos más frecuentes fueron también el dolor y el estreñimiento $(18.1 \%)$ y en cáncer de ovario fue la falta de apetito $(6.2 \%)$. La paciente con $\mathrm{Ca}$ de vulva no reportó ninguno de estos síntomas.

Se realizó la comparación de las variables clínicas continuas entre las pacientes bien nutridas y con riesgo de desnutrición o desnutrición severa obteniendo que la cuantificación de la albúmina fue menor en el grupo de pacientes con riesgo de desnutrición y desnutrición severa, con una media de $3.66 \mathrm{~g}$, en comparación con la presentada por el grupo de bien nutrida, con una media de $4.14 \mathrm{~g}$, siendo estadísticamente significativa (p 0.016). El \% de pérdida de peso en 1 mes (\%PP1m) que presentaron las pacientes, previo a la evaluación nutricional, fue mayor en el grupo de riesgo de desnutrición y desnutrición severa, con una media de $6.24 \%$ vs $0.58 \%$ en las pacientes bien nutridas (p 0.000). La pérdida de peso en 6 meses (PP6m) (peso reportado por las pacientes que presentaron 6 meses antes de la evaluación nutricional) fue mayor en las pacientes con riesgo de desnutrición y con desnutrición severa, (media 9.36\%) en comparación con el grupo de bien nutridas (media 2.67\%) ( $\mathrm{p}$ 0.000). El índice de masa corporal, el porcentaje de grasa y de músculo no presentaron diferencia estadísticamente significativa entre ambas categorías (Tabla 5).

Tabla 5. Estado nutricional evaluado por VGS-GP entre ambas categorías

\begin{tabular}{|c|c|c|c|c|c|}
\hline Grupo & & $\mathrm{N}$ & Media & DS & $\begin{array}{c}\text { Media de } \\
\text { error } \\
\text { estándar }\end{array}$ \\
\hline \multirow{2}{*}{ Edad (años)* } & $A$ & 29 & 47.6 & 11.6 & 2.2 \\
\hline & $\mathrm{B}$ y $\mathrm{C}$ & 35 & 55.7 & 14.2 & 2.4 \\
\hline \multirow{2}{*}{ IMC $(\mathrm{kg} / \mathrm{m} 2)^{*}$} & $A$ & 29 & 31.5 & 6.3 & 1.2 \\
\hline & $B$ y $C$ & 35 & 29.4 & 7.1 & 1.2 \\
\hline Porcentaje de & $A$ & 29 & 43.5 & 7.4 & 1.4 \\
\hline Grasa & $B$ y $C$ & 30 & 42.2 & 8.7 & 1.6 \\
\hline Porcentaje de & $A$ & 29 & 24.4 & 2.8 & 0.5 \\
\hline Músculo* & $B$ y $C$ & 30 & 25.0 & 3.8 & 0.7 \\
\hline \multirow{2}{*}{$\begin{array}{l}\text { Albúmina } \\
\text { (gramos)* }\end{array}$} & $A$ & 22 & 4.1 & 0.4 & 0.1 \\
\hline & $\mathrm{B}$ y $\mathrm{C}$ & 23 & 3.7 & 0.8 & 0.2 \\
\hline \multirow{2}{*}{ PP1m (\%)* } & A & 28 & 0.6 & 1.0 & 0.2 \\
\hline & $B$ y $C$ & 34 & 6.2 & 8.0 & 1.4 \\
\hline \multirow{2}{*}{ PP6m (\%)* } & $A$ & 28 & 2.7 & 4.1 & 0.8 \\
\hline & $\mathrm{B}$ y $\mathrm{C}$ & 35 & 9.4 & 8.4 & 1.4 \\
\hline
\end{tabular}

\section{Discusión}

El tipo de cáncer que predominó en la población estudiada fue cáncer cervicouterino (CaCU) $67.2 \%$, seguido por el cáncer de endometrio (17.2\%), (14\%) cáncer de ovario, (1.6 \%) cáncer de vulva, una 
investigación realizada en Australia utilizó la VGSGP en pacientes con sospecha o diagnóstico de cáncer ginecológico, evaluaron a 194 mujeres de las cuales el $40 \%$ de las pacientes presentó cáncer de endometrio mientras en nuestra investigación fue el $17.2 \%$, el $40 \%$ presentó cáncer de ovario contra el $14 \%$ reportado en nuestro estudio y un $20 \%$ presentó otros tipos de cáncer que incluye cervicouterino, vulva y peritoneal mientras que en nuestro estudio el $1.6 \%$ fue de cáncer de vulva y $67.2 \%$ cáncer cervicouterino siendo este último el más prevalente en las mujeres mexicanas (Laky et al, 2008). Un estudio realizado en Brasil con 146 pacientes con cáncer ginecológico presentó una prevalencia de $58.2 \%$ cáncer cervicouterino, $19.2 \%$ cáncer de endometrio, $17.8 \%$ cáncer de ovario y un $4.5 \%$ de vulva y vaginal, datos similares a los reportados en nuestra investigación (Santos et al, 2015). En India se evaluaron a 60 pacientes con diagnóstico de cáncer ginecológico que visitaban el hospital por primera vez, sin tratamiento para el cáncer, el $40 \%$ de las pacientes presentó cáncer de ovario, $13.3 \%$ cáncer de endometrio, $5 \%$ cáncer de vulva, $1.6 \%$ coriocarcinoma, $1.6 \%$ cáncer vaginal, siendo el diagnóstico más frecuente cáncer de ovario y el de endometrio, obteniendo diferentes prevalencias de diagnósticos en la población de India y la de México (Das et al, 2014).

La Encuesta Nacional de Salud y Nutrición (2012) a nivel nacional reportó que el $1.4 \%$ de las mujeres de 20 a 80 años presentó desnutrición, el $25.2 \%$ presentó peso normal, un $35.5 \%$ presentó sobrepeso y un $37.5 \%$ se diagnosticó con obesidad en base al IMC, de acuerdo a nuestra investigación el $6.3 \%$ presentó (desnutrición), $15.6 \%$ (normopeso), $28.1 \%$ (sobrepeso) y un $50.1 \%$ (obesidad), al comparar ambos datos podemos observar que la desnutrición es mayor en nuestra población de estudio mujeres con diagnóstico de cáncer ginecológico, así como la obesidad (Gutiérrez et al, 2012). En la Encuesta Nacional de Salud y Nutrición (2018-19) un 21.8\% de la población femenina presentó peso normal, un $36.6 \%$ sobrepeso y un $40.2 \%$ obesidad; mientras que en ambos sexos la prevalencia de desnutrición fue de 7.4\% (Shamah-Levy et al., 2020).

Un estudio en pacientes con cáncer ginecológico en Australia reportó que del $100 \%$ de las pacientes con diagnóstico histológico (120 pacientes), el $33.3 \%$ presentó desnutrición (categoría $\mathrm{B}$ y $\mathrm{C}$ ), mientras que en población mexicana un 54.68 \% (categoría B y C) y un $66.3 \%$ del total la población australiana estaba bien nutrido contra un $45.3 \%$ de nuestra población (Laky et al, 2008). Otra investigación realizada en Brasil reportó que el $37.7 \%$ se encontraba bien nutrido (categoría A), el $46.6 \%$ con riesgo de desnutrición (categoría B), y el $15.8 \%$ con desnutrición severa (categoría C) juntos representando el $62.4 \%$ de los pacientes con riesgo de desnutrición/desnutrición severa dato similar al de la población mexicana $54.68 \%$ (Santos et al, 2015). En India reportaron que el $11.6 \%$ de los casos se encontraba bien nutrido (categoría A), $48.3 \%$ tenían riesgo de desnutrición o desnutrición moderada (categoría B) y $40 \%$ estaba en desnutrición severa (categoría $\mathrm{C}$ ), teniendo mayor prevalencia el riesgo de desnutrición/desnutrición severa el $88.3 \%$, dato similar a lo reportado con un $54.68 \%$, no existió diferencia significativa entre los tres grupos (Das et al, 2014).

Santos et al, 2015 en Brasil reportó que el $69.9 \%$ de los pacientes presento algún síntoma gastrointestinal entre ellos el $46.6 \%$ presentó disminución del apetito, $43.8 \%$ náuseas, $31.5 \%$ xerostomía, saciedad temprana $29.5 \%$, disgeusia o ageusia $28.8 \%$, malestar estomacal por olor de los alimentos $27.4 \%$, estreñimiento $26.7 \%$, dolor $23.4 \%$, vómito $23.3 \%$, la presente investigación obtuvo que los signos más frecuentes fueron dolor $32.8 \%$, estreñimiento $31.2 \%$ y falta de apetito $29.6 \%$.

Conforme aumenta el estadio clínico disminuye el porcentaje de pacientes bien nutridas y aumenta el porcentaje riesgo de desnutrición y desnutrición severa, en nuestra investigación de las pacientes en etapa I el 44.8\% se encuentra en "Categoría A - Bien nutrido" mientras un $17.1 \%$ se encuentra en categoría B y C siendo estadísticamente significativo (p 0.0158), similar a la población brasileña donde el $53.8 \%$ de las pacientes bien nutridas se encontraba en estadio I (Santos et al, 2015). De las pacientes en etapa III el $54.3 \%$ se encuentra en riesgo de desnutrición y desnutrición severa y un $27.6 \%$ están bien nutridas (p 0.0313), observando que mientras más avanzado sea el estadio del cáncer existe mayor riesgo de desnutrición, de las pacientes bien nutridas el $44.8 \%$ se encontraban en etapa I de la enfermedad, el $27.6 \%$ en etapa II, $27.6 \%$ en etapa III y no se identificó ninguna en etapa IV. De las pacientes de la categoría B y C el 29.7 \% se encontraban en etapa 
I, $25.7 \%$ en etapa II, 54. $3 \%$ en etapa III y $2.9 \%$ en etapa IV, mientras en de la población estudiada de India el $38.3 \%$ se encontraba en estadio I, un $11.6 \%$ en estadio II, $36.6 \%$ en estadio III y un $13.3 \%$ en estadio IV, siendo más prevalente el estadio III en ambas investigaciones (Das et al, 2014).

La cuantificación de la albúmina fue menor en el grupo de pacientes con riesgo de desnutrición y desnutrición severa con una media de $3.66 \mathrm{~g}$, comparada con el grupo de bien nutridas $4.14 \mathrm{~g}$ (p 0.016); situación similar a la población australiana donde se obtuvo una albúmina sérica disminuida en la población con desnutrición $(3.54 \mathrm{~g} / \mathrm{dl})$ comparado con las bien nutridas $(4.24 \mathrm{~g} / \mathrm{dl})(<0.001)$ (Laky et al, 2008). La pérdida de peso en 1 mes fue mayor en el grupo de riesgo de desnutrición y desnutrición severa, con una media de $6.24 \%$ vs $0.58 \%$ en bien nutridas (p 0.000). La pérdida de peso en 6 meses fue mayor en las pacientes con riesgo de desnutrición y desnutrición severa (media 9.36 \%) en comparación con el grupo de bien nutridas (media $2.67 \%$ ) (p 0.000 ), concluyendo que él \% de pérdida de peso aumenta conforme aumenta el riesgo a desnutrición/desnutrición. En India él \% pérdida de peso aumento conforme mayor era el puntaje de la VGS-GP ( $\mathrm{p}<0.001$ ) (Das et al, 2014). Mientras el índice de masa corporal, el porcentaje de grasa y de músculo no presentaron diferencia estadísticamente significativa entre ambas categorías, situación similar a la reportada por Laky et al, 2008.

Al analizar la clasificación del estado nutricional por el IMC podemos observar que el $50 \%$ tiene obesidad y un $28.1 \%$ tiene sobrepeso; a su vez el $60.9 \%$ de los pacientes presentó un $\%$ de grasa muy alto y un $18.8 \%$ se encontraba en el rango de alto, mientras que el $44.8 \%$ de los pacientes tenía musculo bajo y el resto niveles normales. Es interesante observar cómo aunque existe alta prevalencia de pacientes con obesidad (de acuerdo al IMC y \% de grasa elevados), mientras a la par el $54.68 \%$ presento riesgo a desnutrición o desnutrición severa de acuerdo al tamizaje, lo que nos indica que el paciente con obesidad y cáncer es propenso a tener desnutrición, poco a poco ir perdiendo la masa muscular, desarrollar sintomatología y a su vez mantener o incrementar la grasa corporal, por lo que herramientas como el tamizaje o el \% de pérdida de peso nos pueden ayudar a identificar a pacientes con obesidad y en riesgo de desnutrición, que pueden desarrollar obesidad sarcopenica y presentar alteraciones durante el tratamiento oncológico.

Sabemos que las reservas de masa muscular participan en un papel fundamental para el tratamiento oncológico y la calidad de vida del paciente que vive con cáncer, aunado a las complicaciones inflamatorias que generan el incremento del tejido adiposo, por ello es importante valorar de forma integral al paciente que vive con cáncer, tomar en cuenta la composición corporal y sus modificaciones para establecer una intervención nutricional temprana evitando la pérdida de masa muscular; así como de manera global con el tamizaje empezar a indagar situaciones que pongan en riesgo el estado nutricional y calidad de vida del paciente con cáncer.

\section{Conclusiones}

El 54.68 \% de los pacientes con diagnóstico reciente de cáncer ginecológico tienen riesgo a desnutrición/desnutrición moderada o desnutrición severa de acuerdo con la VGS-GP antes de comenzar cualquier terapia antineoplásica, mientras que el IMC subestima a los pacientes con desnutrición y puede llegar a encubrir el riesgo de desnutrición en pacientes con obesidad de acuerdo al IMC, en los cuales se amerita una intervención nutricional inmediata y eficaz.

Se aprecia como conforme el estadio clínico de la enfermedad aumenta, el \% pérdida de peso aumenta y con ello el riesgo a desnutrición/desnutrición incrementa, a su vez la albúmina disminuye cuando el paciente está en riesgo de desnutrición/desnutrición severa.

Los pacientes con tumores ginecológicos, principalmente aquellos con cáncer cervicouterino y de ovario, estadios II y III de la enfermedad, requieren mayor atención por parte de los profesionales de la salud, ya que están en mayor riesgo nutricional.

La VGS-GP es una herramienta útil y viable para la valoración del estado nutricional en pacientes con cáncer ginecológico, ya que toma en cuenta desde la pérdida de peso reciente, capacidad funcional, ingesta alimentaria, síntomas que disminuyen la ingesta, cambios subjetivos de composición 
corporal, así como factores de estrés propios de la enfermedad que contribuyan a la desnutrición.

Se cumplió el objetivo de determinar la desnutrición en pacientes recién diagnosticadas con cáncer ginecológico con un tamizaje nutricional, una limitación fue el equipo de medición de composición corporal ya que este no es de los más exactos, por lo que se sugiere continuar investigando en esta área.

Nuestros hallazgos respaldan la importancia de la intervención nutricional temprana en la población identificada con riesgo de desnutrición/desnutrición severa, con el objetivo de minimizar la pérdida de masa muscular y mejorar el manejo de los síntomas, ayudando a lograr mejores resultados clínicos.

\section{Agradecimientos}

Al Centro Universitario Contra el Cáncer, UANL por permitirnos realizar la presente investigación.

\section{Bibliografía}

Bauer, J., Capra, S., \& Ferguson, M. (2002). Use of the scored Patient-Generated Subjective Global Assessment (PG-SGA) as a nutrition assessment tool in patients with cancer. European Journal of Clinical Nutrition, 56(8), 779-785. https://doi.org/10.1038/sj.ejcn.1601412

Castillo-Martínez, L., Castro-Eguiluz, D., CopcaekMendoza, E. T., Pérez-Camargo, D. A., ReyesTorres, C. A., Damasco-Ávila, E. A., LópezCórdova, G., Fuentes-Hernández, M. R., CetinaPérez, L., \& Milke-García, M. del P. (2018). Nutritional Assessment Tools for the Identification of Malnutrition and Nutritional Risk Associated with Cancer Treatment. Revista de Investigación Clínica, 70(3), 906. https://doi.org/10.24875/RIC.18002524

Das U., Patel S., Dave K. \& Bhansali R. (2014). Assessment of nutritional status of gynecological cancer cases in India and comparison of subjective and objective nutrition assessment parameters. South Asian Journal of Cancer. Volume 3-Issue (1) DOI: 10.4103/2278$330 X .126518$.

Detsky A., McLaughlin J., Baker J., Johnston N., Whittaker S., Mendelson R. \& Jeejeebhoy K. (1987). What is subjective global assessment of nutritional status? J. Parenter. Enteral Nutr. 11, $8-13$.

Diario Oficial de la Federación. (2014). Reglamento de la Ley General de Salud en Materia de Investigación. Obtenido de: http://www.salud.gob.mx/unidades/cdi/nom/com pi/rlgsmis.html

Frisancho, A. (1981). New norms of upper limb fat and muscles areas for assessment of nutritional status. American Journal of Clinical Nutrition, 34(11), 2540-2545.

Gutiérrez J., Rivera-Dommarco J, Shamah-Levy T, Villalpando-Hernández S, Franco A, CuevasNasu L, Romero-Martínez M, Hernández-Ávila M (2012). Encuesta Nacional de Salud y Nutrición 2012. Resultados Nacionales. Cuernavaca, México: Instituto Nacional de Salud Pública (MX), 2012. ISBN 978-607-511-037-0. Obtenido

de: https://ensanut.insp.mx/informes/ENSANUT201 2ResultadosNacionales.pdf

INEGI (2018). Estadísticas a propósito del día mundial contra el cáncer (4 de febrero). Instituto Nacional de Estadística y Geografía. Obtenido de:

https://www.inegi.org.mx/contenidos/saladepren sa/aproposito/2018/cancer2018_nal.pdf

International Agency for Research on Cancer. (2020). GLOBOCAN 2020: Cancer Today. Obtenido de: http://gco.iarc.fr/today/factsheets-cancers

Jager-Wittenaar, H., \& Ottery, F. D. (2017). Assessing nutritional status in cancer: Role of the Patient-Generated Subjective Global Assessment. Current Opinion in Clinical Nutrition and Metabolic Care, 20(5), 322-329. https://doi.org/10.1097/MCO.000000000000038 9

Kłek, S., Jankowski, M., Kruszewski, W. J., Fijuth, J., Kapała, A., Kabata, P., Rutkowski, P. (2015). Clinical nutrition in oncology: Polish recommendations. Oncology in Clinical Practice, 11(4), 172-188. https://doi.org/10.5603/NJO.2015.0062 
Laky, B., Janda, M., Cleghorn, G., \& Obermair, A. (2008). Comparison of different nutritional assessments and body-composition measurements in detecting malnutrition among gynecologic cancer patients. American Journal of Clinical Nutrition, 87, 1678-1685. https://doi.org/10.3748/wjg.v16.i26.3310.

Marian M. \& Roberts S. (2010). Clinical Nutrition for Oncology Patients. Jones and Bartlett Publishers. Capítulo 2-3. Pp. 21-65.

Martínez Roque, V. R. (2007). Valoración del Estado de Nutrición en el Paciente con Cáncer. Cancerología, 2(22), 315-326.

Ottery, F. D. (1996). Definition of standardized nutritional assessment and interventional pathways in oncology. Nutrition, 12(1), S15S19. 9007(95)00067-4 https://doi.org/10.1016/0899-

Santos Rodrigues, C., \& Villaça Chaves, G. (2015). Patient-Generated Subjective Global Assessment in relation to site, stage of the illness, reason for hospital admission, and mortality in patients with gynecological tumors. Supportive Care in Cancer, 23(3), 871-879. https://doi.org/10.1007/s00520-014-2409-7

Shamah-Levy T, Vielma-Orozco E, HerediaHernández O, Romero-Martínez M, MojicaCuevas J, Cuevas-Nasu L, Santaella-Castell JA, Rivera-Dommarco J. (2020) Encuesta Nacional de Salud y Nutrición 2018-19: Resultados Nacionales. Cuernavaca, México: Instituto Nacional de Salud Pública (MX). ISBN 978-607511-205-3. Obtenido de: https://ensanut.insp.mx/encuestas/ensanut2018/d octos/informes/ensanut_2018_informe_final.pdf

Thompson, K. L., Elliott, L., Fuchs-Tarlovsky, V., Levin, R. M., Voss, A. C., \& Piemonte, T. (2017). Oncology Evidence-Based Nutrition Practice Guideline for Adults. Journal of the Academy of Nutrition and Dietetics, 117(2), 297-310.e47. https://doi.org/10.1016/j.jand.2016.05.010

Valenzuela-Landaeta, K., Rojas, P., \& Basfi-fer, K. (2012). Evaluación nutricional del paciente con cáncer. Nutricion Hospitalaria, 27(2), 516-523. https://doi.org/10.3305/nh.2012.27.2.5525

Vázquez de la Torre, M. J., Stein, K., Vásquez Garibay, E. M., Kumazawa Ichikawa, M. R., Troyo Sanromán, R., Salcedo Flores, A. G., \& Sánchez Zubieta, F. A. (2017). Patient-Generated Subjective Global Assessment of nutritional status in pediatric patients with recent cancer diagnosis. Nutrición Hospitalaria. https://doi.org/10.20960/nh.935

White, J. V., Guenter, P., Jensen, G., Malone, A., \& Schofield, M. (2012). Consensus statement: Academy of nutrition and dietetics and American society for parenteral and enteral nutrition: Characteristics recommended for the identification and documentation of adult malnutrition (undernutrition). Journal of Parenteral and Enteral Nutrition, 36(3), 275283. https://doi.org/10.1177/0148607112440285 